\title{
Anterior Segment Optical Coherence Tomography Indices and Their Value in Diagnosing Corneal Ectasia
}

\author{
Angel Atanassov, Marieta Konareva-Kostianeva, Marin Atanassov \\ Department of Ophthalmology, Faculty of Medicine, Medical University, Plovdiv, Bulgaria \\ Email: marin_aa@abv.bg
}

How to cite this paper: Atanassov, A., Konareva-Kostianeva, M. and Atanassov, M (2021) Anterior Segment Optical Coherence Tomography Indices and Their Value in Diagnosing Corneal Ectasia. Open Journal of Ophthalmology, 11, 313-324.

https://doi.org/10.4236/ojoph.2021.114025

Received: August 18, 2021

Accepted: November 21, 2021

Published: November 24, 2021

Copyright $\odot 2021$ by author(s) and Scientific Research Publishing Inc. This work is licensed under the Creative Commons Attribution International License (CC BY 4.0).

http://creativecommons.org/licenses/by/4.0/

\begin{abstract}
Purpose: To determine the diagnostic value of the anterior segment optical coherence tomography (AS-OCT) indices in differentiating normal from ectatic corneas. Material and Methods: Two groups of patients-with corneal ectasia and normal controls were compared. Each group consists of 80 eyes of 43 age and sex-matched patients. All of them underwent corneal topography with OCULUS Keratograph $5 \mathrm{M}$ and corneal pachymetry with AS-OCT with RTVue-100. The indices generated by the AS-OCT pachymetric scans were analyzed. Results: There was a statistically significant difference for all the examined indices between the two groups with $\mathrm{p}$ values $<0.001$ and a confidence interval of $95 \%$. The minimal corneal thickness (Min) was the best performing index according to the ROC analysis with an area under the curve of 0.976 and a combination of sensitivity and specificity of 0.925 and 0.911 respectively, and a "cut-off" value of 484 microns, followed by the indices of focal thinning-Min-Med and Min-Max with an area under the curve of 0.973 and 0.971 and sensitivity/specificity of $0.938 / 0.962$ and $0.938 / 0.937$ respectively. The rest of the examined parameters had an area under the curve in the range between 0.950 for the central corneal thickness and 0.814 for the outer superior segment. Conclusion: The anterior segment OCT indices showed excellent capability in differentiating ectatic from normal corneas.
\end{abstract}

\section{Keywords}

Keratoconus, Corneal Ectasia, Marginal Pellucid Degeneration, Anterior Segment OCT, Pachymetry

\section{Introduction}

The keratoconus [1] [2] and the marginal pellucid degeneration [3] are the two 
most common corneal ectatic diseases. Both conditions are noninflammatory, bilateral and are characterized by corneal thinning, which leads to protrusion. The diagnostics of the corneal ectasias is based on several clinical features-patient's refractive error, the presence or absence of Vogt striae, Fleisher ring, Munson sign as well as changes in the topographical [4] [5] and the biomechanical properties of the cornea [6] [7] [8] [9].

The main features of keratoconus according to the participants in the latest consensus [10] on the topic are posterior cornea ectasia, abnormal corneal thickness profile and noninflammatory thinning. In contrast to the corneal topography, which provides information about the changes in the anterior surface of the cornea, the corneal tomography and the anterior segment optical coherence tomography are excellent tools for observation of the changes in the corneal properties.

The anterior segment optical coherence tomography (AS-OCT) is already a widespread technology and has an important role in the diagnostic and the follow up of many ocular diseases-corneal dystrophies [11], glaucoma [12] [13] [14], ocular tumors [15] [16], keratitis [17] [18] [19] and others. When it comes to corneal ectasia, the anterior segment OCT gives us an excellent opportunity to evaluate corneal [20] [21] and epithelial [20] [21] thickness.

The purpose of the study is to determine the diagnostic capability of the anterior segment OCT indices in patients with keratoconus and pellucid marginal degeneration.

\section{Patients and Methods}

The current study includes two groups of patients-healthy controls and patients with corneal ectasia, that were age and sex-matched. Each group consists of 80 eyes of 43 caucasian individuals, 23 male and 20 female. Each participant underwent a standard ophthalmological examination followed by corneal topography with OCULUS Keratograph $5 M$ (Oculus, Germany) and anterior-segment OCT with Optovue RTVue-100 (OptoVue, USA). Patients wearing contacts were instructed to discontinue them for at least 3 days before the examination.

Inclusion criteria for the ectatic group are:

- Kmax values above 47 diopters.

- Corneal astigmatism above 3 diopters.

- Asymmetrical topographical map consistent with corneal ectasia. Inclusion criteria for the control group are:

- Kmax values under 47 diopters.

- Symmetrical topographical map consistent with corneal astigmatism. Exclusion criteria for the ectatic group are:

- significant corneal scarring.

- corneal hydrops.

- previous crosslinking.

Exclusion criteria for the control group are: 
- corneal astigmatism greater than 3 diopters.

- myopia or hyperopia greater than 5 diopters.

- history of keratitis.

- ocular surgery.

We took into consideration the following parameters generated by the anterior-segment OCT (Figure 1): Mean corneal thickness in 17 sectors, Min (minimal corneal thickness in the $6 \mathrm{~mm}$ zone), Min-Max index (the maximum corneal thickness subtracted from the minimal corneal thickness), Min-Median index (the mean corneal thickness subtracted from the minimal corneal thickness), SN-IT index (the mean corneal thickness of the interior temporal sector subtracted from the mean corneal thickness of the superior nasal sector), S-I index (the mean corneal thickness of the inferior corneal sector subtracted from the mean corneal thickness of the superior sector).

The statistical analysis was performed using SPSS version 15 and the following tests: Mann-Whitney U test, Independent-Samples T-test and ROC (Receiver
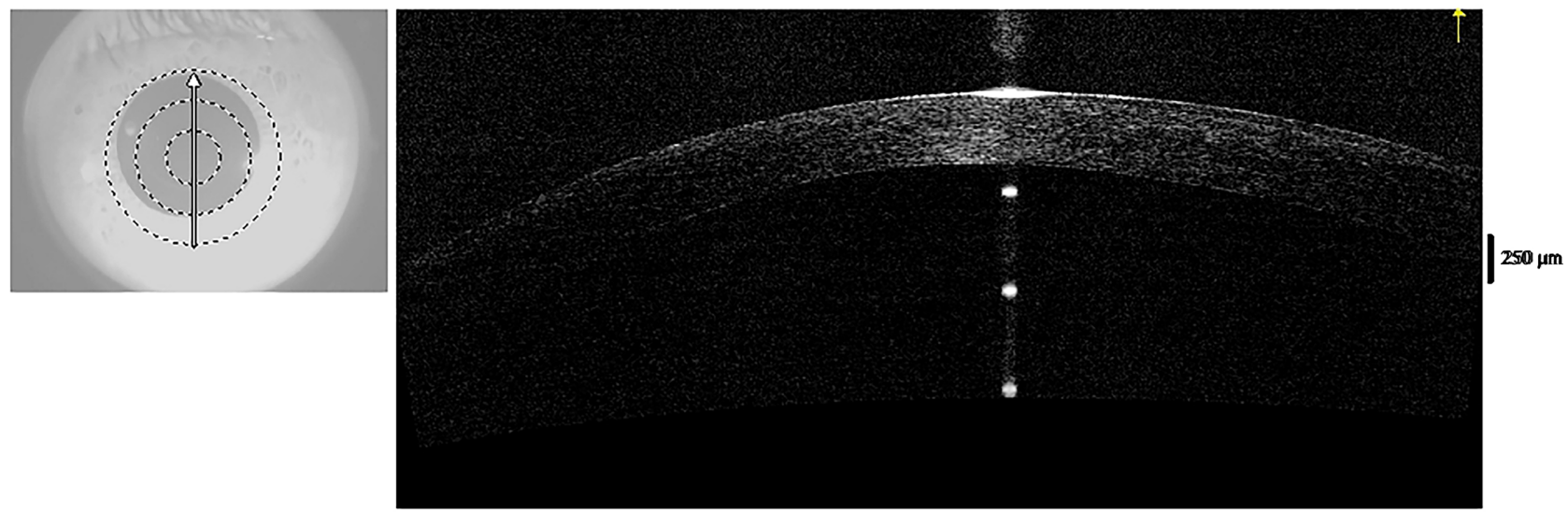

Pachymetry

Pachymetry statistics within central $5 \mathrm{~mm}$ zone

$$
\begin{aligned}
\text { SN-IT }(2-5 \mathrm{~mm}): & 83 & \text { S-I }(2-5 \mathrm{~mm}): & 48 \\
\text { Min: } & 346 & \text { Location Y: } & -578 \\
\text { Min-Median: } & -50 & \text { Min-Max: } & -104
\end{aligned}
$$

Min thickness at $(1.303 \mathrm{~mm},-0.578 \mathrm{~mm})$ indicated as*

Diagnosis:

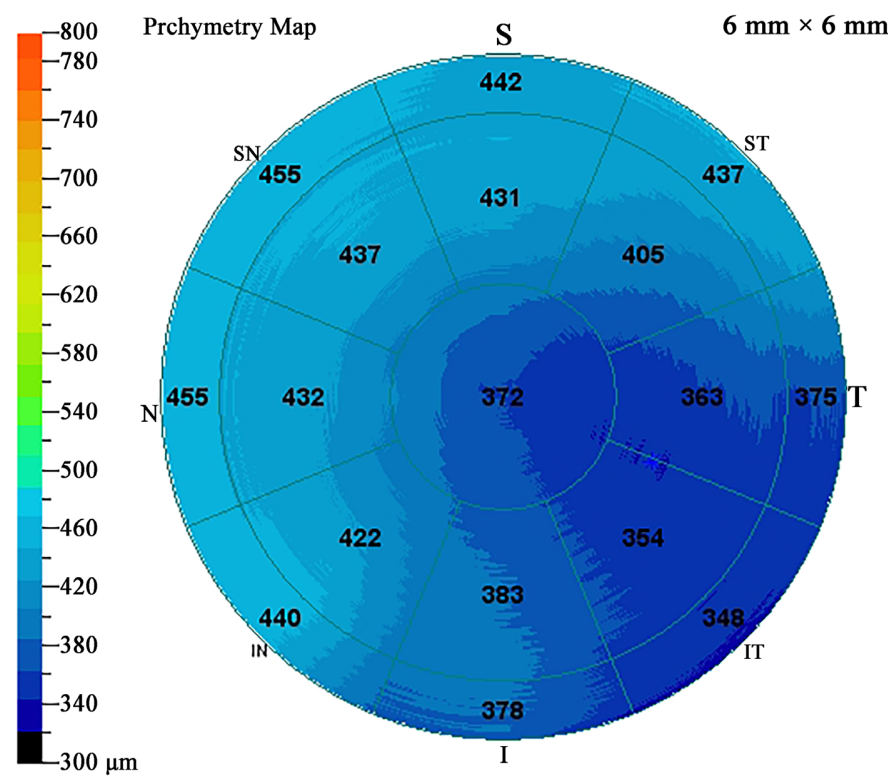

Figure 1. AS-OCT printout with a pachymetric map with corneal thickness in 17 locations. 
Operating Characteristic Analysis).

\section{Results}

The average age in the control group was 34,4 years and 34,6 years in the group with ectasia (Table 1).

After determining the distribution of the data, we used the independent-sample $\mathrm{T}$-test to compare the following indices: central corneal thickness (CCT), superior nasal corneal thickness, inferior nasal corneal thickness, temporal corneal thickness, superior temporal corneal thickness, outer superior nasal corneal thickness, outer nasal corneal thickness, outer inferior nasal corneal thickness, outer inferior temporal corneal thickness, outer temporal corneal thickness, SN-IT and S-N (Table 2).

For the rest of the parameters (superior corneal thickness, nasal corneal thickness, inferior corneal thickness, inferior temporal corneal thickness, outer superior nasal corneal thickness, outer superior temporal corneal thickness, minimal corneal thickness (Min), Min-Max, Min-Med) we used the Mann-Whitney $\mathrm{U}$ test (Table 3). The null hypothesis was rejected for all of the examined indices

Table 1. Age description for the control and ectatic groups

\begin{tabular}{lccccc}
\hline & Average age & St. deviation & Median & Min & Max \\
\hline Control group & 34.4 & 11.4 & 32 & 19 & 66 \\
Ectatic group & 34.6 & 11.6 & 32 & 19 & 66 \\
\hline
\end{tabular}

Table 2. Independent-sample T-test.

\begin{tabular}{cccccccc}
\hline \multirow{2}{*}{ Index } & \multicolumn{2}{c}{ Ectatic group $\mathbf{n}=\mathbf{8 0}$} & \multicolumn{2}{c}{ Control group $\mathbf{n}=\mathbf{8 0}$} & \multirow{2}{*}{ F } & p \\
\cline { 2 - 5 } & Mean & St. dev & Mean & St. dev & & \\
\hline CCT & 447.88 & 41.60 & 533.33 & 32.57 & 4.87 & $<0.001$ \\
SUP NAS & 497.30 & 45.28 & 556.99 & 32.20 & 7.73 & $<0.001$ \\
INF NAS & 472.06 & 47.67 & 551.57 & 34.24 & 6.60 & $<0.001$ \\
TEMP & 452.61 & 44.55 & 537.09 & 31.99 & 5.53 & $<0.001$ \\
SUP TEMP & 480.63 & 42.79 & 546.89 & 31.72 & 5.00 & $<0.001$ \\
O SUP & 518.60 & 44.17 & 563.60 & 31.24 & 5.00 & $<0.001$ \\
O SNAS & 518.09 & 45.42 & 569.32 & 32.79 & 4.67 & $<0.001$ \\
O NAS & 508.85 & 67.45 & 575.10 & 34.78 & 4.97 & $<0.001$ \\
O INAS & 480.86 & 47.34 & 563.80 & 34.09 & 4.83 & $<0.001$ \\
O ITEMP & 454.36 & 48.67 & 548.89 & 33.31 & 7.35 & $<0.001$ \\
O TEMP & 467.30 & 45.34 & 549.84 & 33.11 & 7.38 & $<0.001$ \\
SN-IT & 58.33 & 35.71 & 18.98 & 9.25 & 73.57 & $<0.001$ \\
S-I & 44.08 & 34.87 & 18.98 & 12.42 & 42.41 & $<0.001$ \\
O NAS & 480.86 & 47.34 & 563.38 & 34.09 & 4.83 & $<0.001$ \\
\hline
\end{tabular}


using a confidence interval of $95 \%$ and $p<0.001$, The ROC analysis determined that the mean corneal thickness in several sectors (outer inferior temporal, inferior temporal, central corneal thickness, outer inferior) alongside the indices of focal thinning (Min-Med and Min-Max) had excellent diagnostic capabilities to differentiate ectatic from normal corneas.

On the other hand, thickness asymmetry indices SN-IT and S-I had a lot worse performance (Figure 2).

Table 3. Mann-Whitney U test.

\begin{tabular}{ccccccc}
\hline \multirow{2}{*}{ Index } & \multicolumn{2}{c}{ Ectatic group $\mathbf{n}=\mathbf{8 0}$} & \multicolumn{2}{c}{ Control group $\mathbf{n}=\mathbf{8 0}$} & Mann-Whitney & p \\
\cline { 2 - 5 } & Mean & St. dev & Mean & St. dev & U & \\
\hline SUP & 499.38 & 42.91 & 553.41 & 30.69 & 950.00 & $<0.001$ \\
NAS & 489.48 & 44.32 & 557.92 & 33.91 & 866.00 & $<0.001$ \\
INF & 456.68 & 47.97 & 544.80 & 33.43 & 456.50 & $<0.001$ \\
INF TEMP & 441.26 & 46.35 & 537.82 & 33.07 & 287.50 & $<0.001$ \\
O SUP & 518.60 & 44.17 & 563.60 & 31.24 & 1332.00 & $<0.001$ \\
O ST & 494.53 & 47.07 & 561.80 & 31.75 & 615.50 & $<0.001$ \\
T PACHY & 414.30 & 47.53 & 525.09 & 31.85 & 159.50 & $<0.001$ \\
MIN-MED & -57.20 & 29.20 & -20.06 & 5.51 & 170.00 & $<0.001$ \\
MIN-MAX & -117.56 & 39.16 & -50.00 & 12.27 & 188.00 & $<0.001$ \\
\hline
\end{tabular}

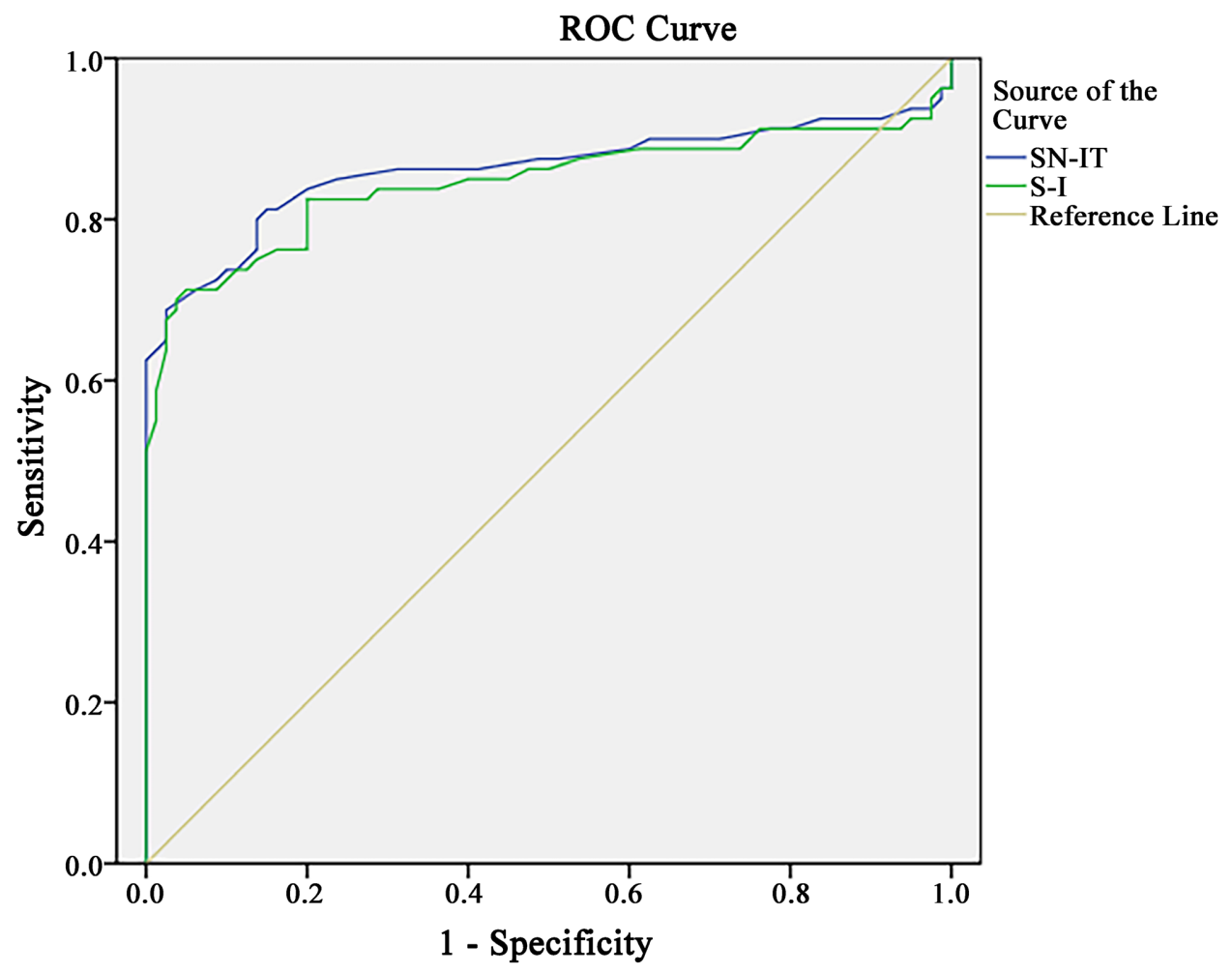

Diagonal segments are produced by ties.

Figure 2. Area under the curve for SN-IT and S-I. 
The minimal corneal thickness (MIN) has the highest area under the curve from all of the examined parameters - 0.976 with a combination of sensitivity and specificity of $0.925,0.911$ and "cut-off" value of 484.5 microns.

Min-Med and Min-Max have an area under the curve of 0.973 and 0.971 respectively (Figure 3). Min-Med index has a sensitivity of 0.938 and specificity of 0.962 with a "Cut off" value of -30 , while the values for the Min-Max index were 0.938/0.937/-68 (sensitivity/specificity/“cut-off” value).

The mean corneal thickness in the outer inferior temporal sector (O INFT) has an area under the curve of 0.956 (Figure 4). The sensitivity, specificity and the "cut-off" values are $0.900,0.886$ and 510.5 respectively. The mean corneal thickness in the inferior temporal sector and the central corneal thickness (Figure 5) have almost identical areas under the curve 0.955 and 0.950.

The combination of sensitivity, specificity and the "cut-off" values are $0.900 /$ $0.900 / 499.5$ for the former and $0.913 / 0.900 / 494.0$ for the latter.

The rest of the sectors with still good performance are the outer inferior (OINF), the temporal (TEMP), the outer temporal (O Temp) and the inferior (INF) one. Their AUC are 0.950, 0.945, 0.939 and 0.927 respectively. The sensitivity, specificity and the "Cut off "values are shown in Table 4.

The area under the curve for the remainder of the indices is in the range from 0.914 to 0.814 .

\section{ROC Curve}

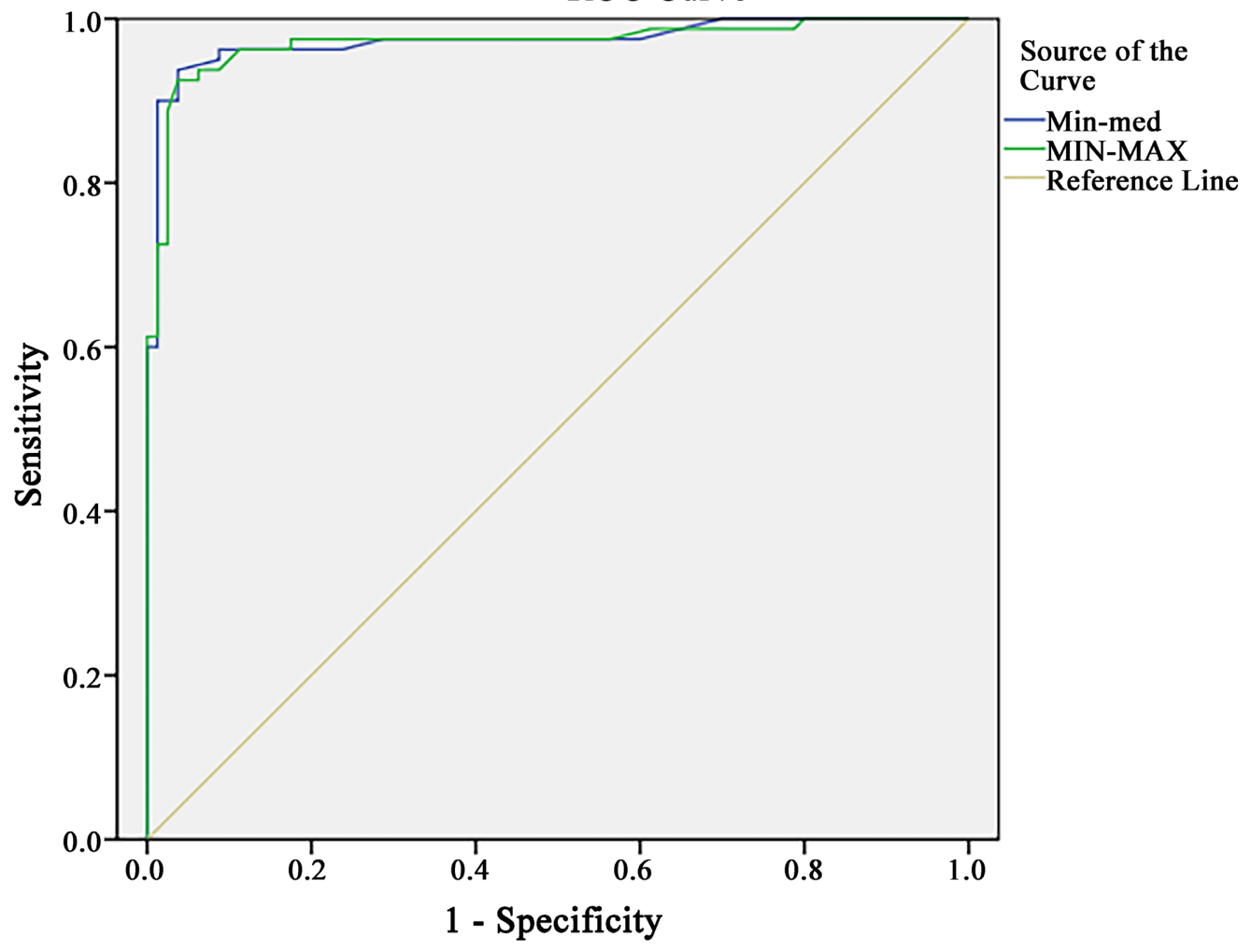

Diagonal segments are produced by ties.

Figure 3. Area under the curve for min-med and min-max. 


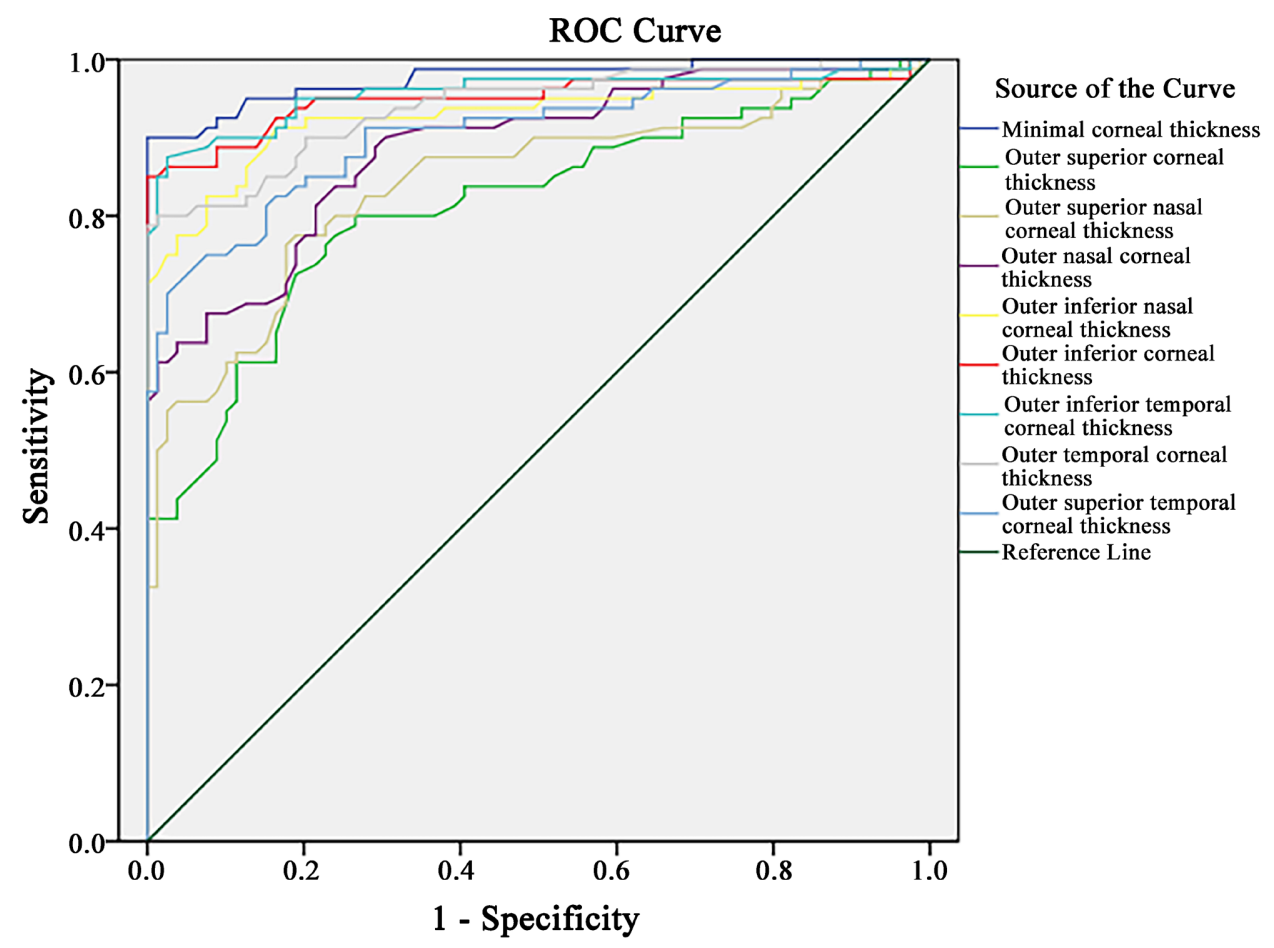

Diagonal segments are produced by ties.

Figure 4. Area under the curve for Minimal corneal thickness and the following corneal sectorsOuter superior, Outer superior nasal, Outer nasal, Outer inferior nasal, Outer inferior, outer inferior temporal, outer temporal, outer superior temporal.

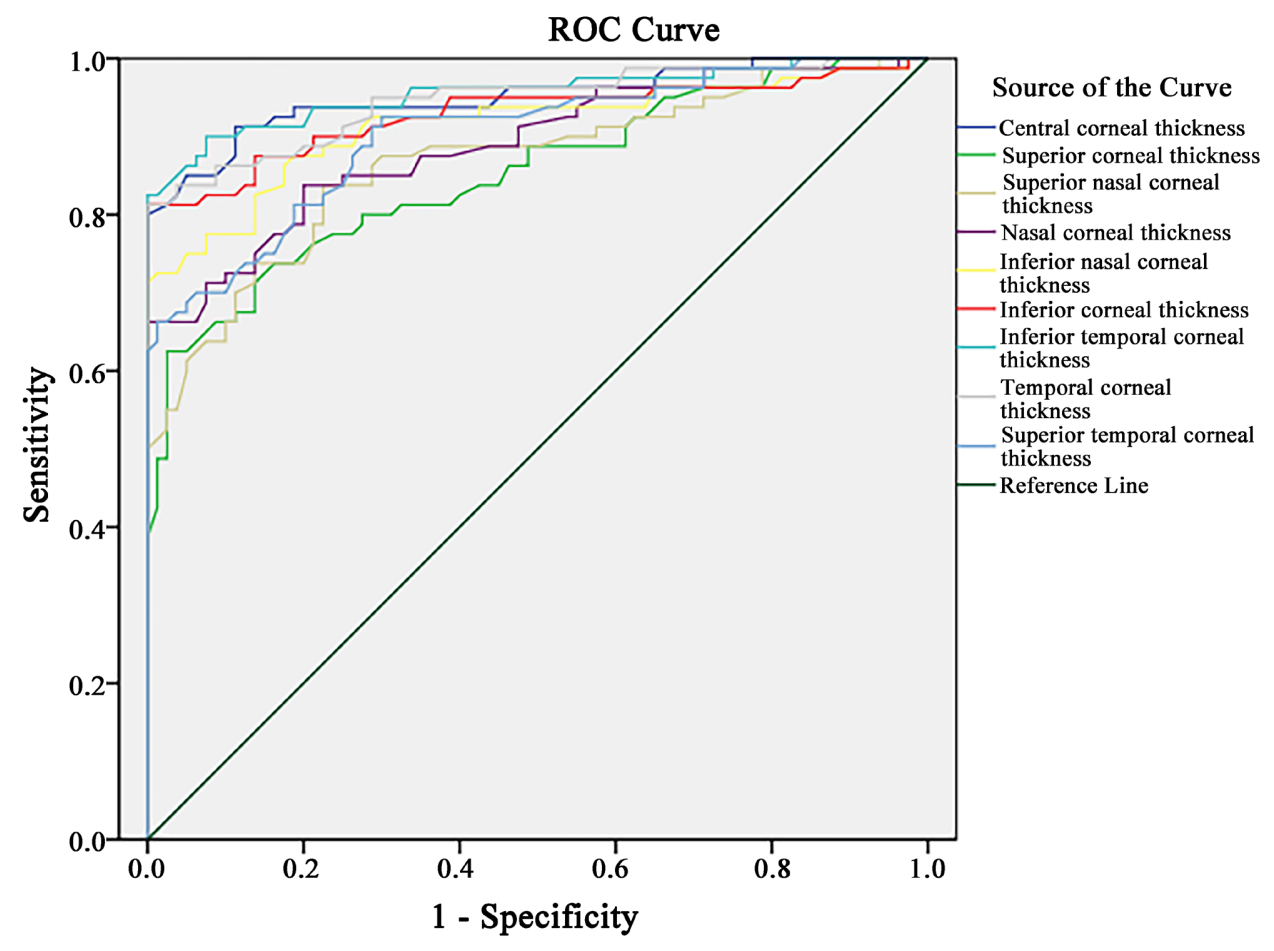

Diagonal segments are produced by ties.

Figure 5. Area under the curve for CCT and the following corneal sectors-superior, superior nasal, nasal, inferior nasal, inferior, temporal, superior temporal. 
Table 4. ROC analysis.

\begin{tabular}{|c|c|c|c|c|c|c|}
\hline \multirow{3}{*}{ Index } & \multirow{3}{*}{ Area } & \multicolumn{2}{|c|}{ Asymptotic $95 \%$} & \multirow{3}{*}{ Cutt off } & \multirow{3}{*}{ Sensitivity } & \multirow{3}{*}{ Specificity } \\
\hline & & \multicolumn{2}{|c|}{ Confidence Interval } & & & \\
\hline & & Lower Bound & Upper Bound & & & \\
\hline MIN & 0.976 & 0.953 & 0.998 & 484.5 & 0.925 & 0.911 \\
\hline Min-med & 0.973 & 0.948 & 0.999 & -30.0 & 0.938 & 0.962 \\
\hline MIN-MAX & 0.971 & 0.944 & 0.998 & -68.0 & 0.938 & 0.937 \\
\hline A INFT & 0.956 & 0.921 & 0.992 & 510.5 & 0.900 & 0.886 \\
\hline INFT & 0.955 & 0.922 & 0.988 & 499.5 & 0.900 & 0.900 \\
\hline CCT & 0.950 & 0.915 & 0.985 & 494.5 & 0.913 & 0.900 \\
\hline A INF & 0.950 & 0.911 & 0.988 & 518.5 & 0.888 & 0.886 \\
\hline TEMP & 0.945 & 0.910 & 0.981 & 507.5 & 0.888 & 0.800 \\
\hline A Temp & 0.939 & 0.903 & 0.976 & 510.5 & 0.900 & 0.886 \\
\hline INF & 0.929 & 0.884 & 0.973 & 513.5 & 0.875 & 0.800 \\
\hline A IN & 0.927 & 0.881 & 0.972 & 531.0 & 0.913 & 0.835 \\
\hline INF N & 0.914 & 0.866 & 0.961 & 528.0 & 0.888 & 0.750 \\
\hline A ST & 0.905 & 0.857 & 0.953 & 510.5 & 0.900 & 0.886 \\
\hline SUPTEMP & 0.902 & 0.855 & 0.949 & 517.5 & 0.813 & 0.812 \\
\hline $\mathrm{N}$ & 0.890 & 0.839 & 0.940 & 531.5 & 0.800 & 0.800 \\
\hline A NAS & 0.886 & 0.835 & 0.937 & 548.5 & 0.775 & 0.797 \\
\hline SUP N & 0.866 & 0.810 & 0.923 & 536.5 & 0.838 & 0.750 \\
\hline SN-IT & 0.861 & 0.796 & 0.927 & 24.5 & 0.838 & 0.800 \\
\hline SUP & 0.852 & 0.792 & 0.911 & 534.5 & 0.788 & 0.725 \\
\hline S-I & 0.845 & 0.777 & 0.913 & 12.5 & 0.913 & 0.775 \\
\hline A SN & 0.841 & 0.778 & 0.904 & 543.0 & 0.775 & 0.797 \\
\hline A SUP & 0.814 & 0.747 & 0.882 & 540.5 & 0.775 & 0.759 \\
\hline
\end{tabular}

\section{Discussion}

In the current study, several indices show excellent diagnostic capabilities.

The central corneal thickness (CCT) and the minimum corneal thickness (Min) are the two best performing parameters with an area under the curve of 0.950 and 0.976 . Before the introduction of the Shleimflug devices and their new indices-ART (Ambrosio relational thickness), PPI (pachymetric progression index), PTI (percentage thickness increase) and others, the CCT [22] [23] [24] and the Min [22] [23] [24] [25] [26] were the most used commonly used pachymetric indices.

There is plenty of publications on the topic, but most of them are based on a tomographic [22] [23] [24] [25] examination and only a few are with ultrasound [27] or OCT devices [26] [27] [28]. Some of the authors haven't taken into consideration the central corneal thickness and for those that have, it is performing worse than MIN [22] [23] [24]. There is a good reason for that-for most of the cases, the zone with the thinning is situated slightly inferior and temporal [27] 
Table 5. Values of indices of different authors.

\begin{tabular}{cccc}
\hline Author & CCT & Min & Device \\
\hline Uçakhan [22] & 0.832 & 0.896 & Pentacam \\
Muftuoglu [23] & 0.832 & 0.873 & Pentacam \\
Mihaltz [24] & 0.880 & 0.900 & Pentacam \\
Ramos [25] & n/a & 0.947 & Pentacam \\
$\operatorname{Li}[26]$ & $\mathrm{n} / \mathrm{a}$ & 0.954 & Zeiss OCT \\
Qin [27] & $\mathrm{n} / \mathrm{a}$ & 0.942 & RTvue OCT \\
\hline
\end{tabular}

and not centrally, so the Min and the CCT value don't match.

Most of the authors also have lower AUC for both CCT and Min (Table 5).

The mean corneal thickness in several sectors also shows promising results. The area under the curve for the following zones: outer inferior, inferior temporal, outer inferior, and temporal, is in the range between 0.956 and 0.945 .

Unfortunately, there are only a few publications with the OptoVueRTVue 100 device, and none of them examined the mean corneal thickness. As already stated, the thinnest zone in patients with ectasia is displaced inferiorly and temporally (in keratoconus) or inferiorly (in PMD), so it makes sense for those sectors to be with the best diagnostic value.

In the current study, the indices of focal thinning (Min-med and Min-max) outperform the thickness asymmetry ones (SN-IT and S-N). The area under the curve for Min-Med is 0.973 and for Min-Max 0.971. In comparison, SN-IT and S-I have a lower area under the curve of 0.861 and 0.843 respectively. Most of the publications on the topic also confirm this finding.

Li et al. [26] were one of the first to use anterior segment OCT in the diagnostics of corneal ectasia. With the Zeiss OCT device, they found AUC for MinMed of 0.929. The I-S and IT-SN indices, which are analogous to the ones we used, also have worse performance when compared to Min-Med with values of 0.868 and 0.894 respectively.

With the RTvue OCT, Qin et al. [28] have reported an AUC of 0.925 for MinMed (they haven't tested Min-Max). The results for SN-IT and S-I are like the ones we have reported -0.896 and 0.861 .

\section{Conclusion}

The anterior segment OCT and its pachymetric indices and maps offer important information about the corneal thickness profile in patients with corneal ectasias. The focal thinning indices alongside the mean corneal thickness in the inferior and the temporal parts of the cornea can be used to distinguish normal from ectatic corneas.

\section{Conflicts of Interest}

The authors declare no conflicts of interest regarding the publication of this paper. 


\section{References}

[1] Krachmer, J.H., Feder, R.S. and Belin, M.W. (1984) Keratoconus and Related Noninflammatory Corneal Thinning Disorders. Survey of Ophthalmology, 28, 293-322. https://doi.org/10.1016/0039-6257(84)90094-8

[2] Rabinowitz, Y.S. (1998) Keratoconus. Survey of Ophthalmology, 42, 297-319. https://doi.org/10.1016/S0039-6257(97)00119-7

[3] Belin, M.W., Asota, I.M., Ambrosio, R. and Khachikian, S.S. (2011) What's in a Name: Keratoconus, Pellucid Marginal Degeneration, and Related Thinning Disorders. American Journal of Ophthalmology, 152, 157-162.E1. https://doi.org/10.1016/j.ajo.2011.03.028

[4] Varssano, D., Kaiserman, I. and Hazarbassanov, R. (2004) Topographic Patterns in Refractive Surgery Candidates. Cornea, 23, 602-607. https://doi.org/10.1097/01.ico.0000121699.74077.f0

[5] Holladay, J.T. (2009) Keratoconus Detection Using Corneal Topography. Journal of Refractive Surgery, 25, S958-S962. https://doi.org/10.3928/1081597X-20090915-11

[6] Vinciguerra, R., Ambrósio, R., Elsheikh, A., Roberts, C.J., Lopes, B., Morenghi, E., et al. (2016) Detection of Keratoconus with a New Biomechanical Index. Journal of Refractive Surgery, 32, 803-811. https://doi.org/10.3928/1081597X-20160629-01

[7] Ali, N.Q., Patel, D.V. and McGhee, C.N.J. (2014) Biomechanical Responses of Healthy and keraToconic Corneas Measured Using a Noncontact ScheimpflugBased Tonometer. Investigative Ophthalmology and Visual Science, 55, 3651-3659. https://doi.org/10.1167/iovs.13-13715

[8] Shah, S., Laiquzzaman, M., Bhojwani, R., Mantry, S. and Cunliffe, I. (2007) Assessment of the Biomechanical Properties of the Cornea with the Ocular Response Analyzer in Normal and Keratoconic Eyes. Investigative Ophthalmology and Visual Science, 48, 3026-3031. https://doi.org/10.1167/iovs.04-0694

[9] Fontes, B.M., Ambrósio, R., Jardim, D., Velarde, G.C. and Nosé, W. (2010) Corneal Biomechanical Metrics and Anterior Segment Parameters in Mild Keratoconus. Ophthalmology, 117, 673-679. https://doi.org/10.1016/j.ophtha.2009.09.023

[10] Gomes, J.A.P., Tan, D., Rapuano, C.J., Belin, M.W., Ambrósio Jr, R., Guell, J.L., et al. (2015) Global Consensus on Keratoconus and Ectatic Diseases. Cornea, 34, 359369. https://doi.org/10.1097/ICO.0000000000000408

[11] Siebelmann, S., Scholz, P., Sonnenschein, S., Bachmann, B., Matthaei, M., Cursiefen, C., et al. (2018) Anterior Segment Optical Coherence Tomography for the Diagnosis of Corneal Dystrophies According to the IC3D Classification. Survey of Ophthalmology, 63, 365-380. https://doi.org/10.1016/j.survophthal.2017.08.001

[12] See, J.L.S., Chew, P.T.K., Smith, S.D., Nolan, W.P., Chan, Y.-H., Huang, D., et al. (2007) Changes in Anterior Segment Morphology in Response to Illumination and after Laser Iridotomy in Asian Eyes: An Anterior Segment OCT Study. British Journal of Ophthalmology, 91, 1485-1489. https://doi.org/10.1136/bjo.2006.113654

[13] Lee, K.S., Sung, K.R., Kang, S.Y., Cho, J.W., Kim, D.Y. and Kook, M.S. (2011) Residual Anterior Chamber Angle Closure in Narrow-Angle Eyes Following Laser Peripheral Iridotomy: Anterior Segment Optical Coherence Tomography Quantitative Study. Japanese Journal of Ophthalmology, 55, 213-219.

[14] Nakano, N., Hangai, M., Nakanishi, H., Inoue, R., Unoki, N., Hirose, F., et al. (2010) Early Trabeculectomy Bleb Walls on Anterior-Segment Optical Coherence Tomography. Graefe's Archive for Clinical and Experimental Ophthalmology, 248, 1173 1182. https://doi.org/10.1007/s00417-010-1311-3 
[15] Krema, H., Santiago, R.A., Gonzalez, J.E. and Pavlin, C.J. (2013) Spectral-Domain Optical Coherence Tomography versus Ultrasound Biomicroscopy for Imaging of Nonpigmented Iris Tumors. American Journal of Ophthalmology, 156, 806-812.E1. https://doi.org/10.1016/j.ajo.2013.05.025

[16] Bianciotto, C., Shields, C.L., Guzman, J.M., Romanelli-Gobbi, M., Mazzuca Jr., D., Ross Green, W., et al. (2011) Assessment of Anterior Segment Tumors with Ultrasound Biomicroscopy versus Anterior Segment Optical Coherence Tomography in 200 Cases. Ophthalmology, 118, 1297-1302. https://doi.org/10.1016/j.ophtha.2010.11.011

[17] Sun, G.H., Li, S.X., Gao, H., Zhang, W.B., Zhang, M.A. and Shi, W.Y. (2012) Clinical Observation of Removal of the Necrotic Corneal Tissue Combined with Conjunctival Flap Covering Surgery under the Guidance of the AS-OCT in Treatment of Fungal Keratitis. International Journal of Ophthalmology, 5, 88-91.

[18] Hixson, A., Blanc, S. and Sowka, J. (2014) Monitoring Keratitis Resolution with Optical Coherence Tomography. Optometry and Vision Science, 91, S40-S45. https://doi.org/10.1097/OPX.0000000000000189

[19] Soliman, W., Fathalla, A.M., El-Sebaity, D.M. and Al-Hussaini, A.K. (2013) Spectral Domain Anterior Segment Optical Coherence Tomography in Microbial Keratitis. Graefe's Archive for Clinical and Experimental Ophthalmology, 251, 549-553. https://doi.org/10.1007/s00417-012-2086-5

[20] Temstet, C., Sandali, O., Bouheraoua, N., Hamiche, T., Galan, A., El Sanharawi, M., et al. (2015) Corneal Epithelial Thickness Mapping Using Fourier-Domain Optical Coherence Tomography for Detection of form Fruste Keratoconus. Journal of Cataract and Refractive Surgery, 41, 812-820. https://doi.org/10.1016/j.jcrs.2014.06.043

[21] Rocha, K.M., Perez-Straziota, C.E., Stulting, R.D. and Randleman, J.B. (2013) SDOCT Analysis of Regional Epithelial Thickness Profiles in Keratoconus, Postoperative Corneal Ectasia, and Normal Eyes. Journal of Refractive Surgery, 29, 173-179. https://doi.org/10.3928/1081597X-20130129-08

[22] Uçakhan, Ö.Ö., Çetinkor, V., Özkan, M. and Kanpolat, A. (2011) Evaluation of Scheimpflug Imaging Parameters in Subclinical Keratoconus, Keratoconus, and Normal Eyes. Journal of Cataract and Refractive Surgery, 37, 1116-1124. https://doi.org/10.1016/j.jcrs.2010.12.049

[23] Muftuoglu, O., Ayar, O., Hurmeric, V., Orucoglu, F. and Kilic, I. (2015) Comparison of Multimetric D Index with Keratometric, Pachymetric, and Posterior Elevation Parameters in Diagnosing Subclinical Keratoconus in Fellow Eyes of Asymmetric Keratoconus Patients. Journal of Cataract and Refractive Surgery, 41, 557-565. https://doi.org/10.1016/j.jcrs.2014.05.052

[24] Miháltz, K., Kovács, I., Takács, Á. and Nagy, Z.Z. (2009) Evaluation of Keratometric, Pachymetric, and Elevation Parameters of Keratoconic Corneas with Pentacam. Cornea, 28, 976-980. https://doi.org/10.1097/ICO.0b013e31819e34de

[25] Ramos, I., Correia, F.F., Lopes, B., Salomão, M.Q. and Correa, R.O. (2012) Topometric and Tomographic Indices for the Diagnosis of Keratoconus. International Journal of Keratoconus and Ectatic Corneal Diseases, 1, 92-99. https://doi.org/10.5005/jp-journals-10025-1018

[26] Li, Y., Meisler, D.M., Tang, M., Lu, A.T.H., Thakrar, V., Reiser, B.J., et al. (2008) Keratoconus Diagnosis with Optical Coherence Tomography Pachymetry Mapping. Ophthalmology, 115, 2159-2166. https://doi.org/10.1016/j.ophtha.2008.08.004

[27] Reinstein, D.Z., Gobbe, M., Archer, T.J., Silverman, R.H. and Coleman, J. (2010) Epithelial, Stromal, and Total Corneal Thickness in Keratoconus: Three-Dimensional Display with Artemis Very-High Frequency Digital Ultrasound. Journal of 
Refractive Surgery, 26, 259-271. https://doi.org/10.3928/1081597X-20100218-01

[28] Qin, B., Chen, S., Brass, R., Li, Y., Tang, M., Zhang, X., et al. (2013) Keratoconus Diagnosis with Optical Coherence Tomography-Based Pachymetric Scoring System. Journal of Cataract and Refractive Surgery, 39, 1864-1871.

https://doi.org/10.1016/j.jcrs.2013.05.048 